\title{
Vertex-Edge Degree Based Indices of Honey Comb Derived Network
}

\author{
Muhammad Ibrahim ${ }^{1, *}$, Sadia Husain ${ }^{2}$, Nida Zahra ${ }^{1}$ and Ali Ahmad ${ }^{2}$ \\ ${ }^{1}$ Centre for Advanced Studies in Pure and Applied Mathematics, Bahauddin Zakariya University, Multan, Pakistan \\ ${ }^{2}$ College of Computer Sciences and Information Technology, Jazan University, Jazan, Saudi Arabia \\ *Corresponding Author: Muhammad Ibrahim. Email: mibtufail@gmail.com \\ Received: 01 March 2021; Accepted: 29 April 2021
}

\begin{abstract}
Chemical graph theory is a branch of mathematics which combines graph theory and chemistry. Chemical reaction network theory is a territory of applied mathematics that endeavors to display the conduct of genuine compound frameworks. It pulled the research community due to its applications in theoretical and organic chemistry since 1960. Additionally, it also increases the interest the mathematicians due to the interesting mathematical structures and problems are involved. The structure of an interconnection network can be represented by a graph. In the network, vertices represent the processor nodes and edges represent the links between the processor nodes. Graph invariants play a vital feature in graph theory and distinguish the structural properties of graphs and networks. In this paper, we determined the newly introduced topological indices namely, first $v e$-degree Zagreb $\alpha$ index, first ve-degree Zagreb $\beta$ index, second ve-degree Zagreb index, ve-degree Randic index, ve-degree atom-bond connectivity index, $v e$-degree geometric-arithmetic index, ve-degree harmonic index and ve-degree sum-connectivity index for honey comb derived network. In the analysis of the quantitative structure property relationships (QSPRs) and the quantitative structureactivity relationships (QSARs), graph invariants are important tools to approximate and predicate the properties of the biological and chemical compounds. Also, we give the numerical and graphical representation of our outcomes.
\end{abstract}

Keywords: Honey comb derived network; ev-degree; topological indices

\section{Introduction}

A structural molecular diagram is a basic diagram in the study of structural chemical graph theory where atoms are spoken to by nodes and chemical bonds are spoken to by lines. A diagram is associated if there is an association between any pair of nodes. A network is an associated diagram that has no various lines between two nodes and loop. The number of nodes which are associated with a fixed node $v$ is known as the degree of $v$ and is denoted by $d_{v}$. The collection of all the adjacent nodes to the node $v$ is referred to open neighborhood of $v$ and can be represented by $N(v)$. The open neighborhood became the closed neighborhood when we include the node $v$ in the collection and is represented by $N[v]$. The shortest distance between two vertices $u, v \in V(G)$ is denoted by $\mathrm{d}(u, v)$, and the maximum value of $\mathrm{d}(u, v)$ in $G$ is called the diameter of $G$, denoted as $\operatorname{diam}(G)$. For basic definition, see West [1].

This work is licensed under a Creative Commons Attribution 4.0 International License, which permits unrestricted use, distribution, and reproduction in any medium, provided the original work is properly cited. 
The relation between the $(Q S P R)$ and $(Q S A R)$ predict the properties and natural exercises of unstudied material. In these materials, the topological indices and some physico-chemical properties are utilized to anticipate bioactivity for chemical compounds [2-5]. A number represents a topological index in a diagram of a chemical compound, which can be utilized to portray the underlined chemical compound and help to foresee its physio-chemical properties. In 1947 Weiner established the framework of topological index. He was approximated the breaking point of alkanes and presented the Weiner index [6]. In 1975, Milan Randic presented Randic index [7]. In 1998, Bollobas et al. [8] and Amic et al. [9] proposed the general Randic index and has been concentrated by both scientist and mathematicians [10]. The Randic index is one of the most important and generally considered and applied topological index. Numerous surveys, papers and books [11-16] are composed on graph invariant. For detail of different topological indices, see [17-22]. Chellali et al. [23] introduced two novel degree thoughts which they called "ve-degree and $e v$-degree". Horoldagva et al. [24] contributed to the study related to "ve-degree and $e v$-degree". The new style degree base indices have been applied to already existing indices and found the better results in [25-27]. It has been found that the ve-degree Zagreb index has more grounded estimate power than the old-style Zagreb index.

\section{The ve-degree and $e v$-degree Based Topological Indices}

Chellali et al. [23] gave the definition of $e v$-degree of an edge $e=u v \in E$ which is denoted by $d_{e v}(e)$, and is the cardinality of nodes of the union of the closed neighborhoods of $u$ and $v$. The ve-degree of the node $v \in V$, denoted by $d_{v e}(v)$, and is the cardinality of lines of different lines that are incident to any node from the closed neighborhood of $v$. Throughout this paper we consider $G$ is a connected graph, $e=u v \in E(G)$ and $v \in V$. For some basic definitions regarding "ev-degree and ve-degree topological indices" [28-30]. The topological indices related to $e v$-degree are: The $e v$-degree Zagreb index, $e v$-degree Randic index, The topological indices related to $e v$-degree are: The first ve-degree Zagreb $\alpha$ index, first ve-degree Zagreb $\beta$ index, second ve-degree Zagreb index, ve-degree Randic index, ve-degree atom-bond connectivity index, $v e$-degree geometric-arithmetic index, ve-degree harmonic index and ve-degree sum-connectivity index.

\section{Main Results}

In the present section, we determined our computational results for Honey Comb derived network (see Fig. 1), which is a planar graph. The number of nodes and lines in $\operatorname{HcDN1}(n)$ are $9 n^{2}-3 n+1$ and $27 n^{2}-21 n+6$ respectively.

There are five types of lines in $H c D N 1(n)$ based on degrees of end nodes of each line. Tab. 1 shows line partition of $H c D N 1(n)$. Tab. 2 represents the number of nodes corresponding their degrees.

In Tab. 3, We partition the lines, based on ev-degree of the HcDN1. In Tabs. 4 and 5, we partition the nodes, based on ve-degree of $H c D N 1$.

\section{Computing Indices for $H c D N 1$ Formulas}

In this section, we will calculate $e v$-degree and ve-degree based indices of the different types of indices which are given as under;

- ev-degree Zagreb Index 


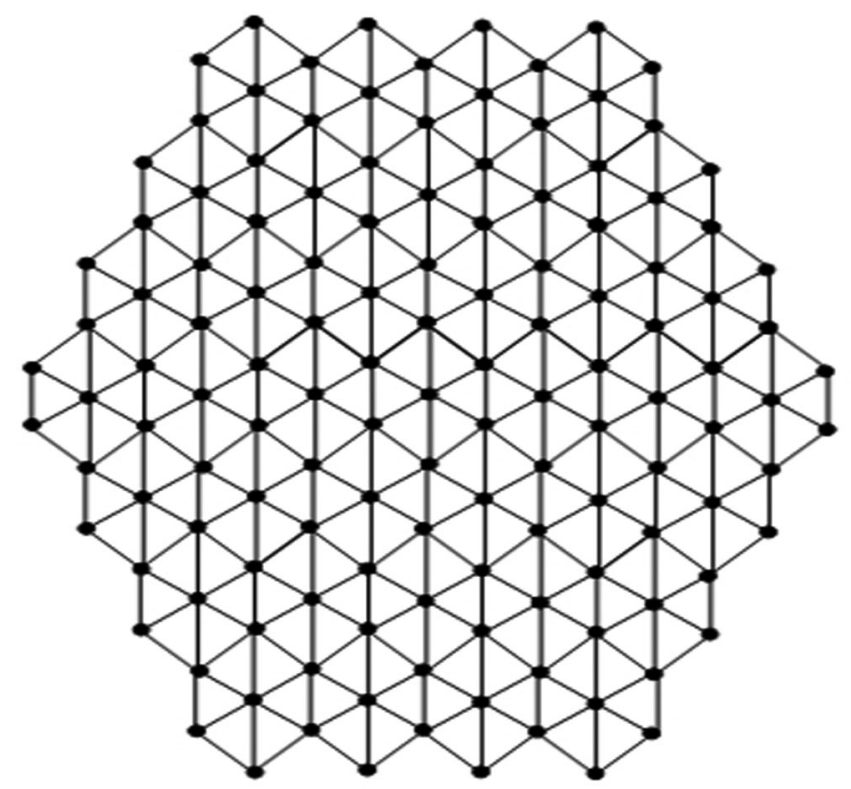

Figure 1: $\operatorname{HcDN} 1(n)$ network with $n=4$

Table 1: Line partition $H c D N 1$

\begin{tabular}{ll}
\hline$(d(u), d(v))$ & Number of lines \\
\hline$(3,3)$ & 6 \\
$(3,5)$ & $12(n-1)$ \\
$(3,6)$ & $6 n$ \\
$(5,6)$ & $18(n-1)$ \\
$(6,6)$ & $27 n^{2}-57 n+30$ \\
\hline
\end{tabular}

Table 2: Number of nodes with corresponding degrees

\begin{tabular}{ll}
\hline$d(u)$ & Number of nodes \\
\hline 3 & $6 n$ \\
5 & $6(n-1)$ \\
6 & $9 n^{2}-15 n+7$ \\
\hline
\end{tabular}

Table 3: Line partition of $H c D N 1$

\begin{tabular}{lll}
\hline Number of lines & Degree of its end nodes & ev-degrees \\
\hline 6 & $(3,3)$ & 6 \\
$12(n-1)$ & $(3,5)$ & 8 \\
$6 n$ & $(3,6)$ & 9 \\
$18(n-1)$ & $(5,6)$ & 11 \\
$27 n^{2}-57 n+30$ & $(6,6)$ & 12 \\
\hline
\end{tabular}


Table 4: Node partition of $H c D N 1$

\begin{tabular}{ll}
\hline Number of nodes & $v e$-degrees \\
\hline 12 & 12 \\
$6(n-2)$ & 14 \\
$6(n-1)$ & 20 \\
6 & 22 \\
$6(n-2)$ & 25 \\
$6(n-1)$ & 29 \\
$9 n^{2}-27 n+19$ & 30 \\
\hline
\end{tabular}

Table 5: The ve-degree of the end nodes of lines of $H c D N 1$

\begin{tabular}{ll}
\hline Number of lines & ve-degrees of its end nodes \\
\hline 6 & $(12,12)$ \\
12 & $(12,20)$ \\
12 & $(12,22)$ \\
12 & $(20,22)$ \\
12 & $(22,29)$ \\
6 & $(29,29)$ \\
$6(3 n-5)$ & $(29,30)$ \\
$6(n-1)$ & $(20,29)$ \\
$12(n-2)$ & $(14,20)$ \\
$6(n-2)$ & $(14,25)$ \\
$12(n-2)$ & $(20,25)$ \\
$12(n-2)$ & $(25,29)$ \\
$6(n-2)$ & $(25,30)$ \\
$27 n^{2}-93 n+78$ & $(30,30)$ \\
\hline
\end{tabular}

Now with the help of Tab. 3, we compute the $e v$-degree based Zagreb index of HcDN1 as:

$$
\begin{aligned}
\mathcal{M}^{e v}(H c D N 1) & =\sum_{e \in E(H c D N 1)} d_{e v}(e)^{2}, \\
\mathcal{M}^{e v}(H c D N 1) & =6 \times 6^{2}+12(n-1) \times 8^{2}+6 n \times 9^{2}+18(n-1) \times 11^{2}+\left(27 n^{2}-57 n+30\right) \times 12^{2} \\
& =216+768 n-768+486 n+2178 n-2178+3888 n^{2}-8208 n+4320 \\
& =3888 n^{2}-4776 n+1590 .
\end{aligned}
$$

- The first ve-degree Zagreb $\alpha$ index 
Now with the help of Tab. 4, we compute the first ve-degree Zagreb $\alpha$ index of $H c D N 1$ as:

$$
\begin{aligned}
\mathcal{M}_{1}^{\alpha v e}(H c D N 1)= & \sum_{v \in V(H c D N 1)} d_{v e}(v)^{2}, \\
\mathcal{M}_{1}^{\alpha v e}(H c D N 1)= & 12 \times 12^{2}+6(n-2) \times 14^{2}+6(n-1) \times 20^{2}+6 \times 22^{2}+6(n-2) \times 25^{2} \\
& +6(n-1) \times 29^{2}+\left(9 n^{2}-27 n+19\right) \times 30^{2} \\
= & 1728+1176 n-2352+2400 n-2400+2904+3750 n-7500 \\
& +5046 n-5046+8100 n^{2}-24300+17100 \\
= & 8100 n^{2}-11928 n+4434 .
\end{aligned}
$$

- The first ve-degree Zagreb $\beta$ index

Now with the help of Tab. 5, we compute the first ve-degree Zagreb $\beta$ index of HcDN1 as:

$$
\begin{aligned}
\mathcal{M}_{1}^{\beta v e}(H c D N 1)= & \sum_{u v \in E(H c D N 1)}\left(d_{v e}(u)+d_{v e}(v)\right), \\
\mathcal{M}_{1}^{\beta v e}(H c D N 1)= & 6 \times 24+12 \times 32+12 \times 34+12 \times 42+12 \times 51+6 \times 58+6(3 n-5) \times 59 \\
& +6(n-1) \times 49+12(n-2) \times 34+6(n-2) \times 39+12(n-2) \times 45+12(n-2) \\
& \times 54+6(n-2) \times 55+\left(27 n^{2}-93 n+78\right) \times 6 \\
= & 144+384+408+504+612+348+1062 n-1770+294+408 n-816+234 n \\
& -468+540 n-1080+648 n-1296+330 n-660+1620 n^{2}-5580 n+4680 \\
= & 1620 n^{2}-2064 n+696 .
\end{aligned}
$$

- The second ve-degree Zagreb index

Now with the help of Tab. 5, we compute the second ve-degree based Zagreb index of HcDN1 as:

$$
\begin{aligned}
\mathcal{M}_{2}^{v e}(H c D N 1)= & \sum_{u v \in E(H c D N 1)}\left(d_{v e}(u) \times d_{v e}(v)\right), \\
\mathcal{M}_{2}^{v e}(H c D N 1)= & 6 \times 144+12 \times 240+12 \times 264+12 \times 440+12 \times 638+6 \times 841+6(3 n-5) \\
& \times 870+6(n-1) \times 580+12(n-2) \times 280+6(n-2) \times 350+12(n-2) \times 500 \\
& +12(n-2) \times 725+6(n-2) \times 750+\left(27 n^{2}-93 n+78\right) \times 900 \\
= & 864+2880+3168+5280+7656+5046+15660 n-26100+3480 n-3480 \\
& +3360 n-6720+2100 n-4200+6000 n-12000+8700 n-17400+4500 n \\
& -9000+24300 n^{2}-83700 n+70200 \\
= & 24300 n^{2}-39900 n+16194 .
\end{aligned}
$$

- The ve-degree Randic index

Now with the help of Tab. 5, we compute the ve-degree Randic index of $H c D N 1$ as:

$$
\begin{aligned}
\mathcal{R}^{v e}(H c D N 1)= & \sum_{u v \in E(H c D N 1)}\left(d_{v e}(u) \times d_{v e}(v)\right)^{-\frac{1}{2}}, \\
\mathcal{R}^{v e}(H c D N 1)= & 6 \times 144^{-\frac{1}{2}}+12 \times 240^{-\frac{1}{2}}+12 \times 264^{-\frac{1}{2}}+12 \times 440^{-\frac{1}{2}}+12 \times 638^{-\frac{1}{2}} \\
& +6 \times 841^{-\frac{1}{2}}+6(3 n-5) \times 870^{-\frac{1}{2}}+6(n-1) \times 580^{-\frac{1}{2}}+12(n-2) \times 280^{-\frac{1}{2}} \\
& +6(n-2) \times 350^{-\frac{1}{2}}+12(n-2) \times 500^{-\frac{1}{2}}+12(n-2) \times 725^{-\frac{1}{2}}+6(n-2) \times 750^{-\frac{1}{2}} \\
& +\left(27 n^{2}-93 n+78\right) \times 900^{-\frac{1}{2}}
\end{aligned}
$$




$$
\begin{aligned}
= & \frac{6}{12}+\frac{12}{4 \sqrt{15}}+\frac{12}{2 \sqrt{66}}+\frac{12}{2 \sqrt{110}}+\frac{12}{\sqrt{638}}+\frac{6}{\sqrt{841}}+\frac{18}{\sqrt{870}} n-\frac{30}{\sqrt{870}}+\frac{6}{2 \sqrt{145}} n-\frac{6}{2 \sqrt{145}}+\frac{12}{2 \sqrt{70}} n \\
& -\frac{24}{2 \sqrt{70}}+\frac{6}{5 \sqrt{14}} n-\frac{12}{5 \sqrt{14}}+\frac{12}{5 \sqrt{20}} n-\frac{24}{5 \sqrt{20}}+\frac{12}{5 \sqrt{29}} n-\frac{24}{5 \sqrt{29}}+\frac{6}{5 \sqrt{30}} n-\frac{12}{5 \sqrt{30}}+\frac{27}{30} n^{2} \\
& -\frac{93}{30} n+\frac{78}{30} \\
= & \frac{9}{10} n^{2}+\left(\frac{18}{\sqrt{870}}+\frac{3}{\sqrt{145}}+\frac{6}{\sqrt{70}}+\frac{6}{5 \sqrt{14}}+\frac{12}{5 \sqrt{20}}+\frac{12}{5 \sqrt{29}}+\frac{6}{5 \sqrt{30}}-\frac{31}{10}\right) n+\frac{1}{2}+\frac{3}{\sqrt{15}}+\frac{6}{\sqrt{66}} \\
& +\frac{6}{\sqrt{110}}+\frac{12}{\sqrt{638}}+\frac{6}{\sqrt{841}}-\frac{30}{\sqrt{870}}-\frac{3}{\sqrt{145}}-\frac{12}{\sqrt{70}}-\frac{12}{5 \sqrt{14}}-\frac{24}{5 \sqrt{20}}-\frac{24}{5 \sqrt{29}}-\frac{12}{5 \sqrt{30}}+\frac{13}{5} \\
= & 0.9 n^{2}-0.0013 n+0.122
\end{aligned}
$$

- The $e v$-degree Randic index

Now with the help of Tab. 3, we compute the ev-degree based Randic index of HcDN1 as:

$$
\begin{aligned}
& \mathcal{R}^{e v}(H c D N 1)=\sum_{e \in E(H c D N 1)} d_{e v}(e)^{-\frac{1}{2}} \\
& \mathcal{R}^{e v}(H c D N 1)=6 \times 6^{-\frac{1}{2}}+12(n-1) \times 6^{-\frac{1}{2}}+6 n \times 9^{-\frac{1}{2}}+18(n-1) \times 11^{-\frac{1}{2}}+\left(27 n^{2}-57 n+30\right) \times 12^{-\frac{1}{2}} \\
& =\frac{27}{\sqrt{12}} n^{2}+\left(\frac{6}{\sqrt{2}}+2+\frac{18}{\sqrt{11}}-\frac{57}{\sqrt{12}}\right) n+\left(\sqrt{6}-\frac{6}{\sqrt{2}}-\frac{18}{\sqrt{11}}+\frac{15}{\sqrt{3}}\right) . \\
& =7.794 n^{2}-4.785+1.44 .
\end{aligned}
$$

- The atom-bond connectivity index

Now with the help of Tab. 5, we compute the atom-bond connectivity index of HcDN1 as:

$$
\begin{aligned}
\mathcal{A B C}^{v e}(H c D N 1)= & \sum_{u v \in E(H c D N 1)} \sqrt{\frac{d_{v e}(u)+d_{v e}(v)-2}{d_{v e}(u) \times d_{v e}(v)}}, \\
\mathcal{A B C}^{v e}(H c D N 1)= & 6 \times \sqrt{\frac{24-2}{144}}+12 \times \sqrt{\frac{32-2}{240}}+12 \times \sqrt{\frac{34-2}{264}}+12 \times \sqrt{\frac{42-2}{440}} \\
& +12 \times \sqrt{\frac{51-2}{638}}+6 \times \sqrt{\frac{58-2}{841}}+6(3 n-5) \times \sqrt{\frac{59-2}{870}}+6(n-1) \times \sqrt{\frac{49-2}{580}} \\
& +12(n-2) \times+6(n-2) \times \sqrt{\frac{39-2}{350}}+12(n-2) \times \sqrt{\frac{45-2}{500}} \\
& +12(n-2) \times \sqrt{\frac{54-2}{725}}+6(n-2) \times \sqrt{\frac{55-2}{750}}+\left(27 n^{2}-93 n+78\right) \times \sqrt{\frac{60-2}{900}}
\end{aligned}
$$




$$
\begin{aligned}
= & \frac{6 \sqrt{22}}{12}+\frac{12 \sqrt{30}}{4 \sqrt{15}}+\frac{48 \sqrt{2}}{2 \sqrt{66}}+\frac{24 \sqrt{10}}{2 \sqrt{110}}+\frac{84}{\sqrt{638}}+\frac{12 \sqrt{14}}{\sqrt{841}}+\frac{18 \sqrt{57}}{\sqrt{870}} n-\frac{30 \sqrt{57}}{\sqrt{870}}+\frac{6 \sqrt{47}}{2 \sqrt{145}} n-\frac{6 \sqrt{47}}{2 \sqrt{145}} \\
& +\frac{48 \sqrt{2}}{2 \sqrt{70}} n-\frac{96 \sqrt{2}}{2 \sqrt{70}}+\frac{6 \sqrt{37}}{5 \sqrt{14}} n-\frac{12 \sqrt{37}}{5 \sqrt{14}}+\frac{12 \sqrt{43}}{5 \sqrt{20}} n-\frac{24 \sqrt{43}}{5 \sqrt{20}}+\frac{24 \sqrt{13}}{5 \sqrt{29}} n-\frac{48 \sqrt{13}}{5 \sqrt{29}}+\frac{6 \sqrt{53}}{5 \sqrt{30}} n \\
& -\frac{12 \sqrt{53}}{5 \sqrt{30}}+\frac{27 \sqrt{58}}{30} n^{2}-\frac{93 \sqrt{58}}{30} n+\frac{78 \sqrt{58}}{30} \\
= & \frac{9 \sqrt{58}}{10} n^{2}+\left(\frac{18 \sqrt{57}}{\sqrt{870}}+\frac{3 \sqrt{47}}{\sqrt{145}}+\frac{24}{\sqrt{35}}+\frac{6 \sqrt{37}}{\sqrt{14}}+\frac{12 \sqrt{43}}{\sqrt{20}}+\frac{24 \sqrt{13}}{5 \sqrt{29}}+\frac{6 \sqrt{53}}{5 \sqrt{30}}-\frac{31 \sqrt{58}}{10}\right) n+\frac{\sqrt{22}}{2} \\
& +3 \sqrt{2}+\frac{24}{\sqrt{33}}+\frac{12}{\sqrt{11}}+\frac{84}{\sqrt{638}}+\frac{12 \sqrt{14}}{\sqrt{841}}-\frac{30 \sqrt{57}}{\sqrt{870}}-\frac{3 \sqrt{47}}{2 \sqrt{145}}-\frac{48}{\sqrt{35}}-\frac{12 \sqrt{37}}{5 \sqrt{14}}-\frac{24 \sqrt{43}}{5 \sqrt{20}}-\frac{48 \sqrt{13}}{5 \sqrt{29}} \\
& -\frac{12 \sqrt{53}}{5 \sqrt{30}}+\frac{13 \sqrt{58}}{5} . \\
= & 6.854 n^{2}+18.92 n+1.855 .
\end{aligned}
$$

- The geometric-arithmetic index

Now with the help of Tab. 5, we compute the geometric-arithmetic index of HcDN1 as:

$$
\begin{aligned}
& \mathcal{G}^{v e}(H c D N 1)=\sum_{u v \in E(H c D N 1)} \frac{2 \sqrt{\operatorname{deg}_{v e}(u) \times d_{v e}(v)}}{d_{v e}(u)+d_{v e}(v)}, \\
& \mathcal{G} \mathcal{A}^{v e}(H c D N 1)=6 \times \frac{2 \sqrt{144}}{24}+12 \times \frac{2 \sqrt{240}}{32}+12 \times \frac{2 \sqrt{264}}{34}+12 \times \frac{2 \sqrt{440}}{42}+12 \times \frac{2 \sqrt{638}}{51} \\
& +6 \times \frac{2 \sqrt{841}}{58}+6(3 n-5) \times \frac{2 \sqrt{870}}{59}+6(n-1) \times \frac{2 \sqrt{580}}{49}+12(n-2) \times \frac{2 \sqrt{280}}{34} \\
& +6(n-2) \times \frac{2 \sqrt{350}}{39}+12(n-2) \times \frac{2 \sqrt{500}}{45}+12(n-2) \times \frac{2 \sqrt{725}}{54} \\
& +6(n-2) \times \frac{2 \sqrt{750}}{55}+\left(27 n^{2}-93 n+78\right) \times \frac{2 \sqrt{900}}{60} \\
& =6+3 \sqrt{15}+\frac{24 \sqrt{66}}{17}+\frac{8 \sqrt{110}}{7}+\frac{8 \sqrt{638}}{17}+\frac{6 \sqrt{841}}{29}+\frac{36 \sqrt{870}}{59} n-\frac{60 \sqrt{870}}{59}+\frac{24 \sqrt{145}}{49} n-\frac{24 \sqrt{145}}{49} \\
& +\frac{24 \sqrt{70}}{17} n-\frac{48 \sqrt{70}}{17}+\frac{20 \sqrt{14}}{13} n-\frac{40 \sqrt{14}}{13}+\frac{8 \sqrt{20}}{3} n-\frac{16 \sqrt{20}}{3}+\frac{20 \sqrt{29}}{9} n-\frac{40 \sqrt{29}}{9}+\frac{12 \sqrt{30}}{11} n \\
& -\frac{24 \sqrt{30}}{11}+27 n^{2}-93 n+78 \\
& =\left(\frac{36 \sqrt{870}}{59}+\frac{24 \sqrt{145}}{49}+\frac{24 \sqrt{70}}{17}+\frac{20 \sqrt{14}}{13}+\frac{8 \sqrt{20}}{3}+\frac{20 \sqrt{29}}{9}+\frac{12 \sqrt{30}}{11}-93\right) n+6+3 \sqrt{15}+\frac{24 \sqrt{66}}{17} \\
& +\frac{8 \sqrt{110}}{7}+\frac{8 \sqrt{638}}{17}+\frac{6 \sqrt{841}}{29}-\frac{60 \sqrt{870}}{59}-\frac{24 \sqrt{145}}{49}-\frac{48 \sqrt{70}}{17}-\frac{40 \sqrt{14}}{13}-\frac{16 \sqrt{20}}{3}-\frac{40 \sqrt{29}}{9}-\frac{24 \sqrt{30}}{11} \\
& +78+27 n^{2} \\
& =27 n^{2}-21.669 n+6.195 \text {. }
\end{aligned}
$$


- The harmonic index

Now with the help of Tab. 5, we compute the Harmonic index of $H c D N 1$ as:

$$
\begin{aligned}
\mathcal{H}^{v e}(H c D N 1)= & \sum_{u v \in E(H c D N 1)} \frac{2}{d_{v e}(u)+d_{v e}(v)}, \\
\mathcal{H}^{v e}(H c D N 1)= & 6 \times \frac{2}{24}+12 \times \frac{2}{32}+12 \times \frac{2}{34}+12 \times \frac{2}{42}+12 \times \frac{2}{51}+6 \times \frac{2}{58}+6(3 n-5) \times \frac{2}{59} \\
& +6(n-1) \times \frac{2}{49}+12(n-2) \times \frac{2}{34}+6(n-2) \times \frac{2}{39}+12(n-2) \times \frac{2}{45} \\
& +12(n-2) \times \frac{2}{54}+6(n-2) \times \frac{2}{55}+\left(27 n^{2}-93 n+78\right) \times \frac{2}{60} \\
= & \frac{1}{2}+\frac{3}{4}+\frac{12}{17}+\frac{4}{7}+\frac{8}{17}+\frac{6}{29}+\frac{36}{59} n-\frac{60}{59}+\frac{12}{49}-\frac{12}{49}+\frac{12}{17} n-\frac{24}{17}+\frac{4}{13} n-\frac{8}{13}+\frac{8}{15} n-\frac{16}{15}+\frac{4}{9} n-\frac{8}{9} \\
& +\frac{12}{55} n-\frac{24}{55}+\frac{9}{10} n^{2}-\frac{31}{10} n+\frac{13}{5} \\
= & 0.90 n^{2}-0.036 n+0.122 .
\end{aligned}
$$

- The sum-connectivity index

Now with the help of Tab. 5, we compute the Sum-connectivity index of HcDN1 as:

$$
\begin{aligned}
& \chi^{v e}(H c D N 1)=\sum_{u v \in E(H c D N 1)}\left(d_{v e}(u)+d_{v e}(v)\right)^{-\frac{1}{2}} \\
& \chi^{v e}(H c D N 1)=6 \times 24^{-\frac{1}{2}}+12 \times 32^{-\frac{1}{2}}+12 \times 34^{-\frac{1}{2}}+12 \times 42^{-\frac{1}{2}}+12 \times 51^{-\frac{1}{2}}+6 \times 58^{-\frac{1}{2}} \\
& +6(3 n-5) \times 59^{-\frac{1}{2}}+6(n-1) \times 49^{-\frac{1}{2}}+12(n-2) \times 34^{-\frac{1}{2}}+6(n-2) \times 39^{-\frac{1}{2}} \\
& +12(n-2) \times 45^{-\frac{1}{2}}+12(n-2) \times 54^{-\frac{1}{2}}+6(n-2) \times 55^{-\frac{1}{2}}+\left(27 n^{2}-93 n+78\right) \times 60^{-\frac{1}{2}} \\
& =\frac{3}{\sqrt{6}}+\frac{3}{\sqrt{2}}+\frac{12}{\sqrt{34}}+\frac{12}{\sqrt{42}}+\frac{12}{\sqrt{51}}+\frac{6}{\sqrt{58}}+\frac{18}{\sqrt{59}} n-\frac{30}{\sqrt{59}}+\frac{6}{\sqrt{7}} n-\frac{6}{7}+\frac{12}{\sqrt{34}} n-\frac{24}{\sqrt{34}}+\frac{6}{\sqrt{39}} n \\
& -\frac{12}{\sqrt{39}}+\frac{4}{\sqrt{5}} n-\frac{8}{\sqrt{5}}+\frac{4}{\sqrt{6}} n-\frac{8}{\sqrt{6}}+\frac{6}{\sqrt{55}} n-\frac{12}{\sqrt{55}}+\frac{27}{2 \sqrt{15}} n^{2}-\frac{93}{2 \sqrt{15}} n+\frac{39}{\sqrt{15}} \\
& =\frac{27}{2 \sqrt{15}} n^{2}+\left(\frac{18}{\sqrt{59}}+\frac{6}{7}+\frac{12}{\sqrt{34}}+\frac{6}{\sqrt{39}}+\frac{4}{\sqrt{5}}+\frac{4}{\sqrt{6}}+\frac{6}{\sqrt{55}}-\frac{93}{2 \sqrt{15}}\right) n+\frac{3}{\sqrt{6}}+\frac{3}{\sqrt{2}}+\frac{12}{\sqrt{34}}+\frac{12}{\sqrt{42}} \\
& +\frac{12}{\sqrt{51}}+\frac{6}{\sqrt{58}}-\frac{30}{\sqrt{59}}-\frac{6}{7}-\frac{24}{\sqrt{34}}-\frac{12}{\sqrt{39}}-\frac{8}{\sqrt{5}}-\frac{8}{\sqrt{6}}-\frac{12}{\sqrt{55}}+\frac{39}{\sqrt{15}} \\
& =3.486 n^{2}-1.556 n+0.531 \text {. }
\end{aligned}
$$

\section{Numerical and Graphical Representation and Discussion}

The $v e$ and $e v$ for ten different types od degree base topological descriptors for the $H c D N 1$ are calculated both numerically and graphically. From Fig. 2 it is clearly noted that the behavior of first Zegreb alpha index, first Zagreb beta index and second Zagreb index is almost same in the increasing direction as the value of $n$ increases while $e v$ Zagreb index value has a very rapid increase with the increase value of $n$. From Fig. 3 it is clearly noted that the behavior of atom bond connectivity index and geometric arithmetic index are almost 
closely increasing with the increase value of $n$ while $e v$ Randic index value has a very rapid increase with the increase value of $n$. The numerical representation of $H c D N 1$ is shown in Tabs. 6-8. The graphical representation of $H c D N 1$ are shown in Figs. 2-4.

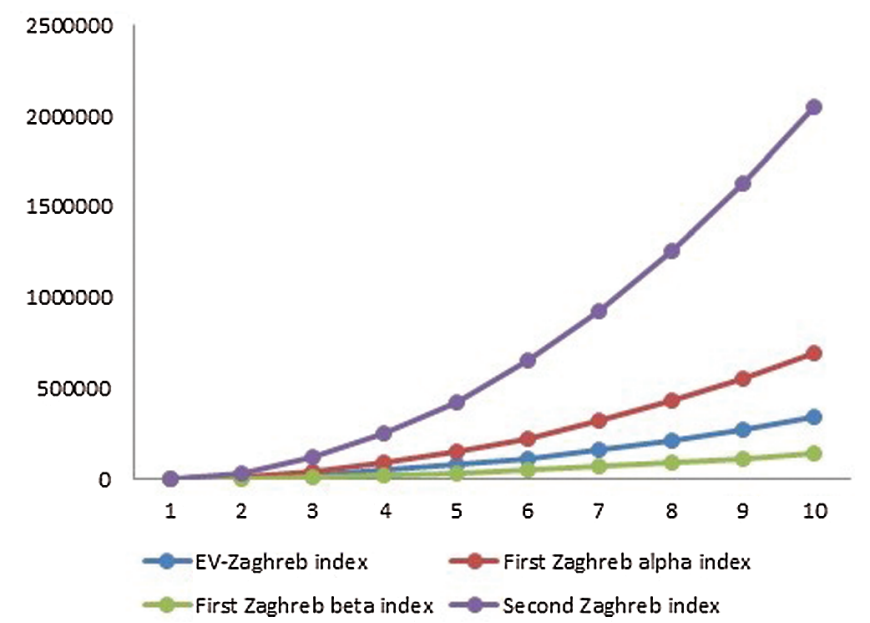

Figure 2: Graphical comparison of $M^{e v}, M_{1}^{\alpha v e}, M_{1}^{\beta v e}$ and $M_{2}^{v e}$

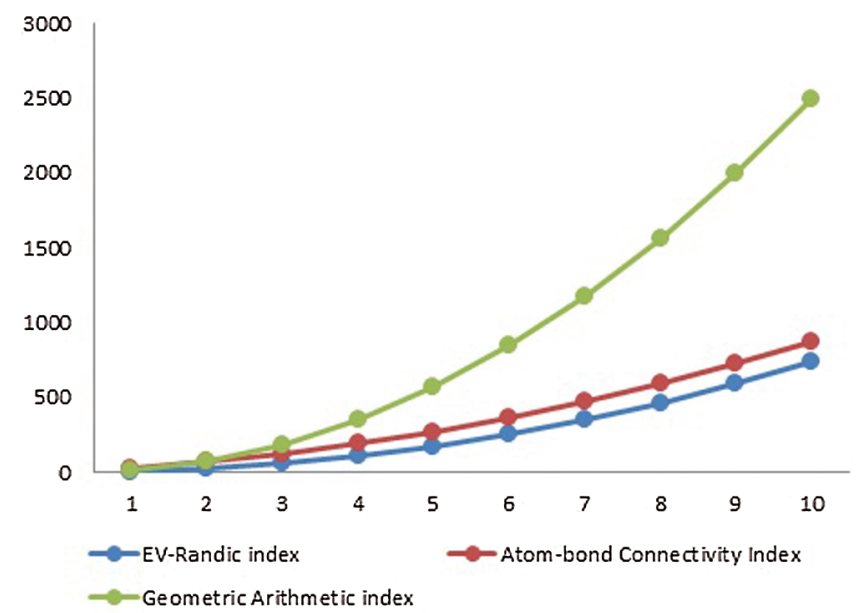

Figure 3: Graphical comparison of $R^{e v}, A B C^{v e}$ and $G A^{v e}$

Table 6: Numerical comparison of $M^{e v}, M_{1}^{\alpha v e}, M_{1}^{\beta v e}$ and $M_{2}^{v e}$

\begin{tabular}{lllll}
\hline $\mathrm{n}$ & $M^{e v}$ & $M_{1}^{\alpha v e}$ & $M_{1}^{\beta v e}$ & $M_{2}^{v e}$ \\
\hline 1 & 702 & 606 & 252 & 594 \\
2 & 7590 & 12978 & 3048 & 33594 \\
3 & 22254 & 41550 & 9084 & 115194 \\
4 & 44694 & 86322 & 18360 & 245394 \\
\hline
\end{tabular}




\begin{tabular}{|c|c|c|c|c|}
\hline $\mathrm{n}$ & $M^{e v}$ & $M_{1}^{\alpha v e}$ & $M_{1}^{\beta v e}$ & $M_{2}^{v e}$ \\
\hline 5 & 74910 & 147294 & 30876 & 424194 \\
\hline 6 & 112902 & 224466 & 46632 & 651594 \\
\hline 7 & 158670 & 317838 & 65628 & 927594 \\
\hline 8 & 212214 & 427410 & 87864 & 1252194 \\
\hline 9 & 273534 & 553182 & 113340 & 1625394 \\
\hline 10 & 342630 & 695154 & 142056 & 2047194 \\
\hline
\end{tabular}

Table 7: Numerical comparison of $R^{e v}, A B C^{v e}$ and $G A^{v e}$

\begin{tabular}{llll}
\hline $\mathrm{n}$ & $R^{e v}$ & $A B C^{v e}$ & $G A^{v e}$ \\
\hline 1 & 4.449 & 27.63 & 11.526 \\
2 & 23.046 & 67.113 & 70.857 \\
3 & 57.231 & 120.304 & 184.188 \\
4 & 107.004 & 187.203 & 351.519 \\
5 & 172.365 & 267.81 & 572.85 \\
6 & 253.314 & 362.125 & 848.181 \\
7 & 349.851 & 470.148 & 1177.512 \\
8 & 461.976 & 591.879 & 1560.843 \\
9 & 589.689 & 727.318 & 1998.174 \\
10 & 732.99 & 876.465 & 2489.505 \\
\hline
\end{tabular}

Table 8: Numerical comparison of $R^{v e}, H^{v e}$ and $\chi^{v e}$

\begin{tabular}{llll}
\hline $\mathrm{n}$ & $R^{v e}$ & $H^{v e}$ & $\chi^{v e}$ \\
\hline 1 & 1.02 & 0.986 & 2.461 \\
2 & 3.719 & 3.65 & 11.363 \\
3 & 8.218 & 8.114 & 27.237 \\
4 & 14.517 & 14.378 & 50.083 \\
5 & 22.615 & 22.442 & 79.901 \\
6 & 32.514 & 32.306 & 116.691 \\
7 & 44.213 & 43.97 & 160.453 \\
8 & 57.116 & 57.434 & 211.187 \\
9 & 73.01 & 72.698 & 268.893 \\
10 & 90.109 & 89.762 & 333.571 \\
\hline
\end{tabular}




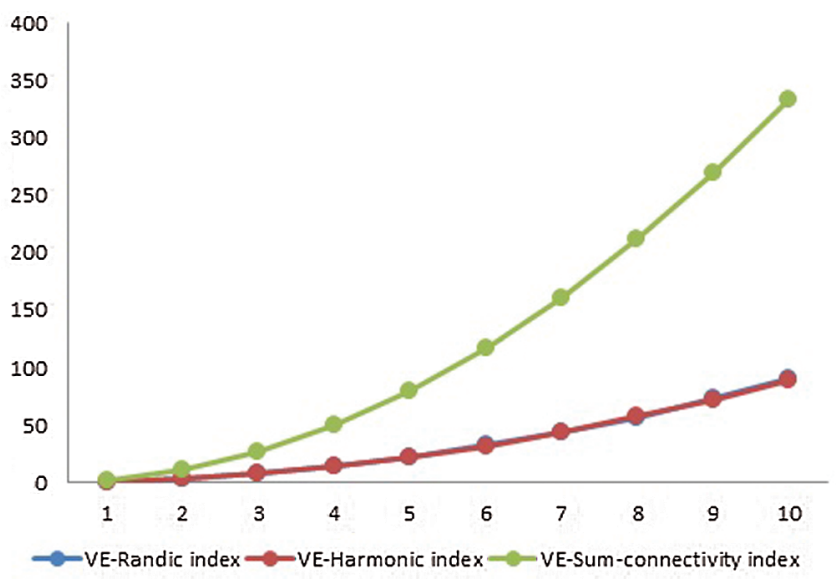

Figure 4: Graphical comparison of $R^{v e}, H^{v e}$ and $\chi^{v e}$

\section{Conclusion}

There are many applications of topological descriptors in computer science, networks, agriculture and chemical graph theory etc. These descriptors help in finding the behavior of their structures. We dealt with the honey comb derived network and computed ten different types of topological descriptors which are base on $e v$ and ve degree. We have computed their explicit formulas and then computed their numerical values for different values of $n$. Further we plotted their graphs for comparison and discussed their behavior. We observe that the values of all descriptors increases with the increase value of $n$. In the analysis of the quantitative structure property relationships (QSPRs) and the quantitative structureactivity relationships (QSARs), graph invariants are important tools to approximate and predicate the properties of the biological and chemical compounds. In this paper, we study the vertex-edge based topological indices for honey comb derived network.

Funding Statement: The authors received no specific funding for this study.

Conflicts of Interest: The authors declare that they have no conflicts of interest to report regarding the present study.

\section{References}

[1] D. B. West, Introduction to graph theory, Second Edition. Pearson Education (Singapore) Pte. Ltd., 2002.

[2] G. Rcker and C. Rcker, "On topological indices, boiling points, and cycloalkanes," Journal of Chemical Information and Computer Sciences, vol. 39, no. 5, pp. 788-802, 1999.

[3] S. Klavzar and I. Gutman, "A comparison of the Schultz molecular topological index with the Wiener index," Journal of Chemical Information and Computer Sciences, vol. 36, no. 5, pp. 1001-1003, 1996.

[4] F. M. Bruckler, T. Doslic, A. Graovac and I. Gutman, "On a class of distance-based molecular structure descriptors," Chemical Physics Letters, vol. 503, no. 4-6, pp. 336-338, 2011.

[5] H. Deng, J. Yang and F. Xia, "A general modeling of some vertex-degree based topological indices in benzenoid systems and phenylenes," Computers and Mathematics with Applications, vol. 61, no. 10, pp. 3017-3023, 2011.

[6] H. Wiener, "Structural determination of paraffin boiling points," Journal of the American Chemical Society, vol. 69, pp. 17-20, 1947.

[7] M. Randic, "Characterization of molecular branching," Journal of the American Chemical Society, vol. 97, no. 23, pp. 6609-6615, 1975.

[8] B. Bollobas and P. Erdos, "Graphs of extremal weights," Ars Combinatoria, vol. 50, pp. 225-233, 1998. 
[9] D. Amic, D. Beslo, B. Lucic, S. Nikolic and N. Trinajstic, "The vertex-connectivity index revisited," Journal of Chemical Information and Computer Sciences, vol. 38, no. 5, pp. 819-822, 1998.

[10] Y. Hu, X. Li, Y. Shi, T. Xu and I. Gutman, "On molecular graphs with smallest and greatest zeroth-order general Randic index," MATCH Communication in Mathematical and in Computer Chemistry, vol. 54, no. 2, pp. 425434, 2005.

[11] X. Li, I. Gutman and M. Randic, "Mathematical aspects of Randic-type molecular structure descriptors," University of Kragujevac and Faculty of Science, Kragujevac, 2006.

[12] M. Randic, "On history of the Randic index and emerging hostility toward chemical graph theory," MATCH Communication in Mathematical and in Computer Chemistry, vol. 59, pp. 5-124, 2008.

[13] M. Randic, "The connectivity index 25 years after," Journal of Molecular Graphics and Modelling, vol. 20, no. 1, pp. 19-35, 2001.

[14] I. Gutman and B. Furtula, "Recent results in the theory of Randic index," University of Kragujevac and Faculty of Science, Kragujevac, 2008.

[15] X. Li and Y. Shi, "A survey on the Randic index," MATCH Communication in Mathematical and in Computer Chemistry, vol. 59, no. 1, pp. 127-156, 2008.

[16] S. Nikolic, G. Kovacevic, A. Milicevic and N. Trinajstic, "The Zagreb indices 30 years after," Croatica Chemica Acta, vol. 76, no. 2, pp. 113-124, 2003.

[17] I. Gutman and K. C. Das, "The first Zagreb index 30 years after," MATCH Communication in Mathematical and in Computer Chemistry, vol. 50, no. 1, pp. 83-92, 2004.

[18] K. C. Das and I. Gutman, "Some properties of the second Zagreb index," MATCH Communication in Mathematical and in Computer Chemistry, vol. 52, pp. 103-112, 2004.

[19] I. Gutman, A. Milicevic, S. Nikolic and N. Trinajstic, "About the Zagreb Indices," Kemija u Industriji, vol. 59, no. 12, pp. 577-589, 2010.

[20] N. Idrees, R. Khalid, F. B. Farooq and S. Nasir, "Computing topological invariants of triangular chandelier lattice," CMC-Computers, Materials \& Continua, vol. 63, no. 3, pp. 1119-1132, 2020.

[21] A. Milicevic, S. Nikolic and N. Trinajstic, "On reformulated Zagreb indices," Molecular Diversity, vol. 8, no. 4, pp. 393-399, 2004.

[22] A. N. A. Koam, A. Ahmad and M. F. Nadeem, "Comparative study of valency-based topological descriptor for hexagon star network," Computer Systems Science and Engineering, vol. 36, no. 2, pp. 293-306, 2021.

[23] M. Chellali, T. W. Haynes, S. T. Hedetniemi and T. M. Lewis, "On degrees and degrees in graphs," Discrete Mathematics, vol. 340, no. 2, pp. 31-38, 2017.

[24] B. Horoldagva, K. C. Das and T. A. Selenge, "On ve-degree and ev-degree of graphs," Discrete Optimization, vol. 31, pp. 1-7, 2019.

[25] S. Ediz, "A new tool for QSPR researches: ev-degree Randi index," Celal Bayar University Journal of Science, vol. 13, no. 3, pp. 615-618, 2017.

[26] B. Sahin and S. Ediz, "On ev-degree and ve-degree topological indices," Iranian Journal of Mathematical Chemistry, vol. 9, no. 4, pp. 263-277, 2018.

[27] S. Ediz, "On ve-degree molecular topological properties of silicate and oxygen networks," International Journal of Computing Science and Mathematics, vol. 9, no. 1, pp. 1-12, 2018.

[28] M. Cancan, "On ev-degree and ve-degree topological properties of Tickysim spiking neural network," Computational Intelligence and Neuroscience, Article ID 8429120, 2019.

[29] A. Ahmad, "Comparative study of ve-degree and $e v$-degree topological descriptors for benzene ring embedded in P-type-surface in 2D network," Polycyclic Aromatic Compounds, 2020.

[30] M. Cancan, "On harmonic and $e v$-degree molecular topological properties of DOX, RTOX and DSL networks," CMC-Computers, Materials \& Continua, vol. 59, no. 3, pp. 777-786, 2019. 